\section{Impacto del recurso humano al implantar un sistema de gestión de inocuidad alimentaria en pequeñas y medianas empresas en Panamá}

\author{
Damarys Cortés 1 (iD), Juana Ramos Chue de \\ Pérez ${ }^{2}$ (D), Wedleys Tejedor 3 (iD) \\ Universidad Tecnológica de Panamá \\ 1damarys.cortes; 2juana.ramos; ${ }^{3}$ wedleys.tejedor\{@utp.ac.pa\} \\ DOI https://doi.org/10.33412/pri.v11.1.2531
}

\section{(2)(1) $\$(0)$}

Resumen: Con el fin de encontrar una alternativa eficiente para la implantación de un sistema de gestión de inocuidad alimentaria (SGIA) que sea afín a las pequeñas y medianas empresas (pymes) de alimentos de nuestro país, se realizó un estudio de caso y una encuesta a 100 pymes de alimentos en Panamá. Se propone un modelo metodológico usando tres pilares básicos: conceptos mínimos de la gestión del recurso humano, una herramienta de la ingeniería de proyectos y las buenas prácticas higiénicas (BPH) como parte de todo SGIA. Se presenta el desarrollo del primer pilar, en el cual se puntualiza el concepto de cultura de inocuidad como un componente clave de los planes estratégicos de implantación de sistemas de gestión. Como parte del modelo, se evaluaron los principales retos que confronta el personal y se identificaron los requisitos mínimos con que debe contar un Departamento de Recursos Humanos, los cuales, una vez adecuados permiten a las empresas alcanzar el éxito en materia de calidad e inocuidad.

Palabras claves: gestión de inocuidad alimentaria, pequeñas y medianas empresas en Panamá, gestión del recurso humano, buenas prácticas higiénicas.

Title: Impact of human resources when implementing a food safety management system in small and medium-sized panamaniam companies

\footnotetext{
Abstract: In order to find an efficient alternative for the implementation of a food safety management system (FSMS) that is related to the small and medium-sized food enterprises (SMFEs) of our country, a case study and a survey of 100 SMFEs in Panama. A methodological model is proposed using three basic pillars: minimum concepts of human resource management, a project engineering tool and good hygienic practices (GHP) as part of all FSMS. The development of the first pillar is presented, in
}

which the concept of safety culture is pointed out as a key component of the strategic plans for implementing management systems. As part of the model, the main challenges facing the staff were evaluated and the minimum requirements that a Human Resources Department must have were identified, which, once adequate, allow companies to achieve success in terms of quality and safety.

Key words: food safety management, small and medium sized Panamanian companies, human resource management, good hygienic practices.

Tipo de artículo: original

Fecha de recepción: 20 de agosto de 2019

Fecha de aceptación: 21 de enero de 2020

\section{Introducción}

La presente investigación es de tipo aplicada; el alcance se sustenta en el uso de una metodología que servirá de base para el desarrollo de un proyecto de implantación de un Sistema de gestión de inocuidad alimentaria (SGIA), orientado a mejorar los estándares en la agroindustria de alimentos panameña. Se cuenta con el apoyo de una empresa de condimentos y especias del distrito de Panamá, lo cual permite que se realice el estudio, que da como resultado un modelo base que a diferencia de los métodos actuales de implantación, pone de relieve la importancia de la cultura de inocuidad como complemento importante para que perdure con el tiempo; siendo que, en algunos de los incidentes de inocuidad alimentaria en los últimos años, la cultura organizacional ha desempeñado un papel importante.

La cultura de inocuidad alimentaria se puede visualizar en términos de cómo y qué piensan los colaboradores en una empresa u organización al respecto; es decir, son las conductas sobre inocuidad alimentaria que practican y demuestran rutinariamente [1] .

Aunque se observa que la agroindustria alimentaria panameña representa el $84 \%$ del producto interno bruto (PIB) total agroindustria [2], faltan políticas de inocuidad cónsonas con la realidad en que vivimos. Todos los países necesitan contar con programas de control de alimentos para garantizar que los suministros nacionales sean inocuos, de buena calidad y estén disponibles en cantidades adecuadas y a precios asequibles, para asegurar que todos los grupos de la población puedan gozar de un estado de salud y nutrición aceptable [3]. Esto indica que el desarrollo de un método eficaz de implantación de un Sistema de gestión de inocuidad (SGI), que contribuya a la resolución de problemas típicos del sector, será un aporte de vital importancia para las pymes de alimentos, así como para sus clientes y consumidores. Convirtiéndose en beneficiarias directas las empresas agroalimentarias, específicamente en el área de inocuidad, ya que al ser inspeccionadas por el Ministerio de Salud (MINSA) facilitaría la gestión y desarrollo de los Programas de prerrequisitos (PPR).

Conforme al Códex Alimentarius, inocuidad es: "La garantía de que un alimento no causará daño al consumidor cuando el 
mismo sea preparado o ingerido de acuerdo con el uso a que se destine" [4].

Para lograr alimentos inocuos se requiere de un enfoque integrado en cada eslabón de la cadena, que comparta responsabilidades especificas, lo que incluye asegurar la inocuidad en cada fase de producción; lo cual inicia desde la materia prima en la finca, la elaboración y almacenamiento, hasta la distribución final. En este proceso, son responsables desde el proveedor, procesador, así como los entes encargados del gobierno, industrias, universidades, comercio, finalizando con los consumidores. Por lo tanto, el éxito de las empresas depende de la rapidez en la búsqueda de soluciones a problemas técnicos, particularmente en fallas presentadas en la inocuidad de los productos.

Implantar el Sistema de análisis de peligros y puntos críticos de control (HACCP), por sus siglas en inglés, es una tarea difícil para las pequeñas y medianas empresas, cuánto más retador se convierten los SGIA, que tienen un alcance mucho mayor [5].

Indica Dessler G. [6], que: Muchos administradores triunfaron porque tuvieron la habilidad para contratar a los individuos adecuados para los puestos correctos, y porque supieron motivar, evaluar y desarrollar a esas personas. Los propietarios y gestores de las empresas alimentarias, especialmente en las más pequeñas, manifiestan una cierta resistencia a cambiar la cultura de su negocio, forma de trabajar y no encuentran razones suficientes para el cambio [7].

Todo Sistema de gestión de inocuidad (SGI) debe contar con los PPR del Sistema HACCP, lo que implica grandes inversiones de tiempo y dinero. Con el fin de minimizar esta inversión de recursos en la implantación de los PPR en un Sistema de gestión de inocuidad y, considerando que en Panamá no se conocen modelos concretos que faciliten la labor de las empresas de alimentos en cuanto a la implantación de los SGIA, se realiza esta investigación, cuyo objetivo es proponer un modelo en el cual se apliquen los principios de mejora continua, identificando la raíz de los problemas, para así definir las acciones preventivas y correctivas.

\section{Antecedentes}

Después de la Ley 66 de 1947 [8], conocida como El Código Sanitario, donde en su libro cuarto que trata sobre Política Sanitaria y Saneamiento, en sus artículos del 183 al 186, se establecen los controles sanitarios al cual son sujetos los alimentos de cualquier naturaleza; surgen los Decretos Ejecutivos 65 del año 1997 [9], por el cual se reglamenta la aplicación del Sistema de análisis de peligros y puntos críticos de control (HACCP) y en adelante se actualiza con los Decretos 352 y el 81 [10], [11], respectivamente; último decreto que modifica los procedimientos estandarizados de las operaciones de limpieza y desinfección, las buenas prácticas de manufactura (BPM) y el HACCP en las plantas que procesan alimentos diversos, y deben contar obligatoriamente con una descripción detallada de los programas de prerrequisitos debidamente implementados.

En la actualidad, se estableció el Decreto 1784 del 2014 [12], que integra el Reglamento técnico centroamericano para las industrias de bebidas y alimentos procesados, así como los
Decretos 352 y 81 , siendo su enfoque en el cumplimiento de las Buenas prácticas de manufactura (BPM).

En el año 2012, la Comisión nacional consultiva de calidad e inocuidad de alimentos [13], elaboró la guía técnica, cuyo objetivo fue el facilitar a los agroindustriales procesadores de alimentos, la elaboración del Manual de BPM-Procedimientos estandarizados de saneamiento siguiendo el reglamento más actualizado en su momento 352 y 81 . Dicha comisión estaba conformada por instituciones gubernamentales, así como la Universidad de Panamá y Universidad Tecnológica de Panamá, por la Agencia Española para la Cooperación Internacional y el Desarrollo (AECID) y por el Ministerio de Comercio e Industrias.

Entre los años 1993 al 2014, basado en las observaciones de la autora principal, se infieren patrones del recurso humano en unas 35 empresas de alimento en Panamá, que incluyeron productos vegetales empacados al vacío, queserías, derivados de la molinería, entre otras, con un recurso humano y tecnológico diverso, sugiriendo que la forma tradicional para la implantación de los POES y PPR no favorecían que la misma fuera sostenible en el tiempo. A partir de esta observación, se propone este estudio con el interés de focalizarlo en una pyme de alimentos, así como en la elaboración de una encuesta a nivel nacional. La empresa seleccionada tiene como principal actividad, la elaboración de fórmulas para la industria cárnica panameña, la misma ha desarrollado una extensa gama de mezclas de aditivos, especias y condimentos totalmente equilibrados, que le permiten a sus clientes obtener una extensa variedad de productos, con sus particularidades de sabor, color, y tecnología de fabricación.

Se consideró una pyme de alimentos panameña, procesadora de especias y condimentos, debido al cumplimiento de los siguientes requisitos preestablecidos. Esta empresa tiene una particularidad especial, ya que entre sus clientes se encuentran grandes industrias cárnicas, que elaboran productos muy sensitivos en materia de inocuidad alimentaria. Además, los clientes de esta empresa procesadora de condimentos son industrias exigentes en el cumplimiento de lineamientos preestablecidos, auditándolos con una frecuencia importante. A su vez, estas grandes industrias (sus clientes) son sujetas a frecuentes auditorías en donde se les exigen altos estándares de calidad e inocuidad, solicitados por las franquicias transnacionales.

Esta interrelación ejerce indirectamente una alta presión hacia esta pyme de condimentos, ya que las industrias transnacionales sin ser necesariamente clientes directos de la empresa solicitan a las grandes corporaciones el cumplimiento de PPR robustos e integrados entre sí por un Sistema de gestión de inocuidad, y estas grandes empresas solicitan elevados estándares a esta pyme de condimentos.

\section{Metodología}

Para este proyecto de investigación se utilizaron tres componentes: Estudio de caso de tipo exploratorio, encuesta en Panamá tomando en cuenta la interrelación entre las variables establecidas y la triangulación metodológica.

La triangulación metodológica es secuencial, puesto que el resultado de estudio de caso sustentó la planeación para la 
aplicación de la encuesta, complementando la información obtenida.

\subsection{Estudio de caso}

El presente estudio se inició en el año 2014, por un periodo de 4 años, con una investigación cualitativa, con base en un estudio de caso, aplicando el análisis temático como técnica de obtención de información. La observación directa y participativa fue realizada en el transcurso de todo el estudio, así como también se realizaron entrevistas detalladas, especialmente a los actores de la empresa seleccionada; logrando identificar y comprender los roles de cada miembro de la organización, mediante la observación de patrones de relación y reconociendo que el conocimiento se construye tomando en cuenta la participación de los diferentes colaboradores de la empresa.

Al respecto, se realizaron entrevistas a la gerente de operaciones y al jefe de control de calidad. El principal motivo por el cual el estudio de caso se realizó en una sola empresa fue debido a su complejidad, por tratarse de una empresa de alimentos donde convergen factores internos y externos que requieren de un análisis exhaustivo; además, se determinó que posteriormente se realizaría una encuesta a las pymes de alimento panameñas a nivel nacional que igualmente debían cumplir con los requisitos.

\subsubsection{Requisitos de la empresa óptima}

- Ser pyme de alimentos panameña.

- Contar con un responsable de la calidad en la empresa, basado en los Decretos Ejecutivos 81 del 2003, 352 del 2001, así como el Decreto 1784 del 2014.

- Empresa legamente establecida con permiso de operaciones vigente.

- Disponibilidad de tiempo para reuniones y coordinación de cada fase del proyecto.

- Compromiso de permitir auditorías e inspecciones del Sistema de gestión de inocuidad.

- Confidencialidad entre las partes.

- Interés en el proceso de mejora continua sobre su SGIA.

\subsubsection{Entrevista a la Gerencia de operaciones}

Se diseñó un formato-encuesta utilizando preguntas abiertas, dirigidas a la alta gerencia de la empresa. Antes de realizar dicha entrevista, sus preguntas fueron validadas con dos Ingenieros en alimentos.

\subsubsection{Instrumento para auditoría diagnóstica}

Para el diseño del instrumento diagnóstico se consultaron diferentes fuentes, dirigidas a la realización de auditorías internas a empresas de alimentos y sus sistemas de gestión de inocuidad alimentaria. Entre los lineamientos consultados están la Norma FSSC 22000:2014. Se encuentran los Decretos de Panamá n. ${ }^{\circ} 352$ del 2001 y el n. ${ }^{\circ} 81$ del 2003 . Además, se analizó el Decreto n. ${ }^{\circ} 1784$ de 2014 que trata sobre "Buenas prácticas de manufactura para la industria de alimentos y bebidas procesados, del Ministerio de Salud de Panamá".
Después de establecer comparaciones y realizar un cuidadoso estudio de estas regulaciones, tanto de las plantas de alimentos, así como de las pymes se elaboró un modelo de formato para la Auditoría diagnóstica.

\subsubsection{Auditoría Diagnóstica}

El principal objetivo de realizar la auditoría diagnóstica consistió en tener un punto de partida en cuanto a la situación inicial de los procesos, incluyendo aspectos, tanto administrativos como de recursos humanos, técnicos y, en especial del Sistema de gestión de calidad e inocuidad, para así establecer prioridades para el diseño de la ruta a seguir en el planteamiento del diseño metodológico base.

Esta auditoría diagnóstica fue interrelacionada con la entrevista realizada a la gerente de operaciones, todo esto con el fin de seleccionar el PPR que serviría como base para el desarrollo del modelo metodológico. El formato utilizado contempla todas las áreas de proceso, los PPR y el Sistema HACCP, así como también el estatus de las instalaciones y aspectos de gestión y recurso humano.

\subsubsection{Selección del Programa de prerrequisitos (PPR)}

Se seleccionó el PPR en base a la auditoría diagnóstica, entrevistas a la gerente de operaciones y observaciones directas al personal, utilizando a la vez un formulario de inspección. El objetivo de las observaciones fue identificar si se cumplían las BPM, como aspecto básico de un SGIA.

Fueron 3 PPR que pudieron servir de base, pues obtuvieron un valor menor al resto de los demás. Estos fueron:

- Mejoramiento continuo, $72 \%$;

- Capacitación de los trabajadores, $50 \%$

- Buenas prácticas de manufactura, $66 \%$

El criterio tomado en consenso fue:

- Se seleccionará el PPR que afecte en mayor proporción los demás procesos, siendo las Buenas prácticas higiénicas como parte de las BPM, el PPR a estudiar.

\subsubsection{Gestión del recurso humano}

Una vez se realiza la Auditoría interna diagnóstica, se identifica el componente de la gestión humana del estudio. Entre los aspectos consultados en el instrumento para la realización de la auditoría interna, se encuentran los relacionados con la capacitación del personal, así como el procedimiento de inducción del recurso humano. Se desarrolla entonces un Programa anual de capacitación del personal, y desde un inicio, se procede a brindar un entrenamiento al recurso humano, incluyendo al personal administrativo. El método utilizado para el entrenamiento al personal fue de tipo lúdico, profundizando en el concepto de cultura de inocuidad, alternativa identificada en artículos de Inglaterra [14], Chile-ACHIPIA (Agencia chilena para la inocuidad y calidad alimentaria), 2018 [15], [16] y Estados Unidos [17].

\subsection{Aplicación de la encuesta a las pymes de alimento panameñas}

El personal encuestador estaba conformado, tanto por estudiantes de Ingeniería en alimentos, como por profesionales 
del área, incluyendo a la autora principal. Posterior a la capacitación el equipo encuestador y a la validación del instrumento, se aplicaron las encuestas a las pymes de alimentos en 7 provincias del país, tal y como se muestra en la tabla 2.

\subsubsection{Población}

Para la selección de la muestra, estos son los requisitos:

- Ser pyme de alimentos en Panamá.

- Contar con un responsable de la calidad en la empresa.

- Basado en los Decretos Ejecutivos 81 del 2003, 352 del 2001, así como el Decreto 1784 del 2014. (Decretos 81; 352, 1784).

- Ser una empresa legamente establecida con permiso de operaciones vigente y confidencialidad entre las partes.

Se utilizó la fórmula 1 [18] para el cálculo del tamaño de la muestra para una población finita. El diseño utilizado para escoger la muestra fue estratificado, utilizando como estrato el tamaño de la pyme (pequeña y mediana), luego los tipos de actividades agroindustriales. La muestra obtenida fue de 100 pequeñas y medianas empresas de alimentos a nivel nacional, clasificadas como pymes, de un total de 2,035 [19], con un error máximo admisible (d) del $8 \%$ y un nivel de confianza de $90 \%$. Se aplicó la encuesta a un total de 100 pymes procesadoras de alimentos para complementar el estudio. Se utilizó este valor d y nivel de confianza, por la aplicación de la estadística inferencial. Esto permite alcanzar un compromiso tomando en cuenta el tiempo e inversión de recursos por la naturaleza del estudio; y de acuerdo a la literatura se establece por convención común en las Ciencias Sociales intervalos de confianza de 90, 95 y 99 por ciento [20].

$$
n=\left\lceil\frac{N * Z_{a}{ }^{2} * p * q}{d^{2} *(N-1)+Z_{a}{ }^{2} * p * q}\right\rceil
$$

En donde:

$Z$ = Desviación del valor medio que se acepta para lograr el nivel de confianza deseado, $90 \%$.

$\mathrm{N}=$ Total de elementos de una población finita.

$p=$ Proporción estimada de la población que presenta concordancia con la legislación alimentaria (cumplimiento del manual de Buenas prácticas de manufactura, entre otros).

$\mathrm{q}=$ Probabilidad de fracaso.

$\mathrm{n}=$ Número de elementos de la muestra.

$d^{2}=$ Precisión (error máximo admisible en términos de proporción.

Debido a que la encuesta es de múltiples preguntas, tanto dicotómicas (Sí, No) como de escala Likert, se realizó el análisis con base en las preguntas que requirieran un tamaño de muestra mayor, así todas estarían cubiertas; tomándose en cuenta que las preguntas dicotómicas requieren un mayor tamaño de muestra que las continuas. El procedimiento consistió en seleccionar para cada variable, con mayor tamaño de muestra, recodificándola entonces de escala Likert a escala Dicotómica.

Se realiza el cálculo del coeficiente de $\alpha$ de Cronbach. Este coeficiente nos permite tener una estimación de la fiabilidad de una escala aditiva formada por varios indicadores cuyo promedio o combinación lineal representará los niveles de un constructo 0 concepto [21] . Para la consulta sobre el cumplimiento del manual de las BPM en las pymes encuestas, se calculan el 2 de Cronbach (0.97), favoreciendo la fiabilidad de la escala de medida utilizada en el presente estudio, por estar arriba de 0.70 , sin embargo, se trata de estimaciones de juicio prudencial.

\subsection{Triangulación metodológica}

Se ha seleccionado la triangulación metodológica como estrategia de investigación; pues según un estudio realizado [22], la triangulación aporta validez y fiabilidad al proceso, resultados y conclusiones, ofrece enriquecimiento y profundidad a la investigación, además es excelente para la eliminación de sesgos. Unido a esto, la triangulación también flexibiliza el diseño de la investigación, puesto que permite que se adapte mejor a los objetivos que se plantea [22].

Una vez realizado el estudio de caso de la pyme de condimentos panameña, mediante una auditoría diagnóstica, entrevistas a la gerente de operaciones, observaciones directas al personal, e inspecciones se realiza una encuesta a unas 100 pymes de alimentos en Panamá y revisión bibliográfica. Posterior a lo cual se aplica la triangulación metodológica, fortaleciendo el impacto del estudio.

\section{Resultados y discusión}

Se identifica que las pymes de alimentos en Panamá confrontan retos importantes para implantar $y$, sobre todo, mantenerse en el proceso de mejora continua.

El presente proyecto de investigación generó como resultado:

- Requisitos mínimos del recurso humano.

- Capacitación lúdica del personal.

- Caracterización de las pymes de alimentos en Panamá, en relación con la inocuidad.

En la tabla 1 se observan los procedimientos y programas seleccionados como imprescindibles para que una pyme de alimentos favorezca el proceso de implantación de un SGIA y se mantenga con el tiempo.

\subsection{Requisitos mínimos del recurso humano}

Para la elaboración de la tabla 1 , donde se describen los requisitos mínimos del recurso humano, se tomaron en cuenta las observaciones directas, entrevistas y evaluaciones post capacitación del personal de la empresa de estudio de caso. Además, a través de la encuesta a las 100 pymes de alimentos, donde 7 cuestionamientos de los 11 presentados, estuvieron dirigidos al recurso humano. Se presentan a continuación los aspectos más relevantes que sugirieron la importancia de que el personal técnico estuviera respaldado por un departamento de personal o del recurso humano para facilitar la implementación de las BPM y otros aspectos de calidad e inocuidad.

Se cuestionó la existencia de un departamento de recursos humanos en la empresa a lo cual respondieron el $65 \%$ de las pymes encuestadas que no, mientras que el $35 \%$ de las 100 pymes, sí poseía un departamento de recursos humanos.

Por otro lado, El $20 \%$ de las pymes contestó que realiza capacitación respecto a las relaciones interpersonales; contestando el $80 \%$ que no. 
En un $50 \%$ de las pymes se encargan de realizar la descripción del puesto; pagar la planilla y capacitación del personal; en un $45 \%$ se realiza la inducción y/o reinducción del personal; en un $35 \%$ se evalúa el desempeño y se realiza el procedimiento de contratación de personal.

Aún sin contar con un departamento de recursos humanos, se presentan requisitos mínimos con los cuales se propone que estas pymes adecúen estos procedimientos básicos para alcanzar el éxito. Los datos antes presentados sirvieron de insumo para el establecimiento de estos requisitos mínimos.

Tabla 1. Requisitos mínimos del recurso humano

\begin{tabular}{|c|c|}
\hline $\mathbf{N}^{\circ}$ & Procedimiento, programa \\
\hline 1 & Procedimiento de contratación de personal \\
\hline 2 & Procedimiento de inducción y reinducción \\
\hline 3 & Procedimiento de descripción del puesto \\
\hline 4 & Procedimiento de evaluación del desempeño \\
\hline 5 & Programa anual de capacitación lúdica \\
\hline 6 & Programa anual de incentivos al personal \\
\hline
\end{tabular}

\subsection{Capacitación lúdica del personal}

Devaki [23], afirma que las actividades lúdicas hacen referencia a situaciones de aprendizaje donde el componente lúdico, de entusiasmo, juega un papel importante. Son formas motoras jugadas. La capacitación es una estrategia para la empresa y más si se basa en su propia cultura de inocuidad.

Al respecto, la autora aplicó en la empresa de estudio, el concepto de capacitación lúdica, donde los colaboradores establecieron nuevas relaciones con sus compañeros, quienes se coordinaron mejor para trabajar en equipo, llegando a acuerdos entre los colaboradores nuevos y los trabajadores de más de 10 años de trabajo, favoreciendo el cumplimiento de las BPM.

Se usaron técnicas como: Taller de cuerdas, herramientas para la solución de problemas (gráfica de causa y efecto). Se realizó una feria de aprendizaje-enseñanza, donde los colaboradores comprendieron más a fondo los procesos y a los clientes internos, pues trabajaron integralemente entre todos.

\subsection{Caracterización de las pymes de alimentos panameñas en relación con la inocuidad}

En la tabla 2, se presentan las ubicaciones de las pymes procesadoras de alimento. Se puede observar que 56 de las 100 empresas pertenecian a la capital, mientras que 44 pymes provenían del interior del país, información útil para su análisis.

Tabla 2. Número de encuestas aplicadas por provincia

\begin{tabular}{|c|c|c|}
\hline Provincia & $\mathbf{n}^{\circ}$ de encuestas aplicadas & Porcentaje \\
\hline Panamá & 56 & $56 \%$ \\
\hline Herrera & 16 & $16 \%$ \\
\hline Chiriquí & 14 & $14 \%$ \\
\hline Los Santos & 7 & $7 \%$ \\
\hline Veraguas & 4 & $4 \%$ \\
\hline Coclé & 2 & $2 \%$ \\
\hline Panamá O. & 1 & $1 \%$ \\
\hline
\end{tabular}

\subsection{Rubros de las pymes agroindustriales evaluadas}

En la tabla 3 se muestra el número de pymes estudiadas según las ramas agroindustriales, basadas en clasificación industrial internacional uniforme de todas las actividades económicas [24], debido a la particularidad de las pymes que fueron estudiadas. Se puede observar que se evaluaron agroindustrias de elaboración de bebidas (10\%), productos de molinería $(7 \%)$, lácteos $(17 \%)$, chocolatería $(3 \%)$, productos cárnicos, pescado, frutas, hortalizas, vegetales, grasas y aceites $(31 \%)$, panadería $(12 \%)$, y por último otros alimentos $(20 \%)$. Esto permitió identificar el comportamiento en cuanto a inocuidad de los alimentos en diferentes empresas.

Tabla 3. Rubros de las pymes agroindustriales evaluadas

\begin{tabular}{|l|c|}
\hline & $\begin{array}{c}\text { Cantidad de pymes } \\
\text { agroindustriales }\end{array}$ \\
\hline Elaboración de bebidas & 10 \\
\hline Elaboración de productos de molinería & 7 \\
\hline Elaboración de productos lácteos & 17 \\
\hline Elaboración de chocolate & 3 \\
\hline $\begin{array}{l}\text { Producción, elaboración, conservación de carne, } \\
\text { pescado, frutas, legumbres, hortalizas, aceites y } \\
\text { grasas }\end{array}$ & 31 \\
\hline Productos de panadería & 12 \\
\hline Elaboración de otros productos alimenticios & 20 \\
\hline
\end{tabular}

\subsection{Nivel tecnológico en las pymes encuestadas}

Como se puede observar en la figura 1, de las 100 pymes evaluadas, el $74 \%$ posee un nivel tecnológico semi-mecanizado, mientras que el $21 \%$ emplea un nivel manual de producción y el $5 \%$ un nivel totalmente mecanizado.

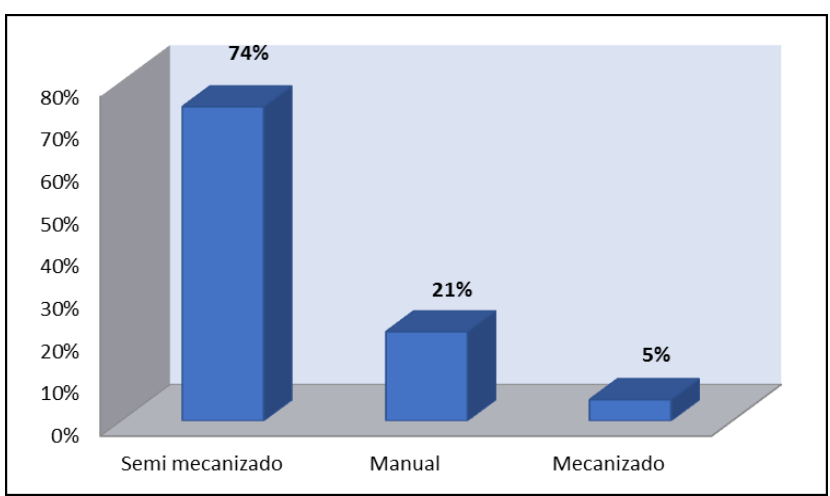

Figura 1. Nivel tecnológico utilizado en las pymes de alimentos

\subsection{Clasificación de las pymes evaluadas, según el número de empleados}

En Panamá, una de las clasificaciones de las pymes, según el Ministerio de Trabajo, está basada en el número de empleados, es decir, según la clasificación de la Organización Internacional del Trabajo (OIT) [25], en la cual las empresas se clasifican en micro (1-9 empleados); pequeña (10-49 empleados); mediana (50-199 empleados) y grande (>200 empleados).

Con esta clasificación, el $53 \%$ de las pymes agroalimentarias evaluadas en este estudio eran pequeñas empresas, mientras 
que el $47 \%$ eran medianas empresas. Esto permitió identificar, tanto en la capital del país como en las demás provincias, la situación actual en que se encuentra su SGIA.

Se presenta en la figura 2, los problemas que con mayor frecuencia enfrentan con el recurso humano en las pymes encuestadas. Se observa que uno de los principales retos es la falta de compromiso del personal (36\%); la falta de capacitación del personal se mencionó como uno de los puntos de importancia (34\%); así como la falta de personal $(29 \%)$. También se indicó que el temor a contratar personal no adecuado era uno de sus retos (24\%); así como la falta de manejo de beneficios (19\%), y la falta de actualización de expedientes con información de las capacitaciones recibidas (10\%).

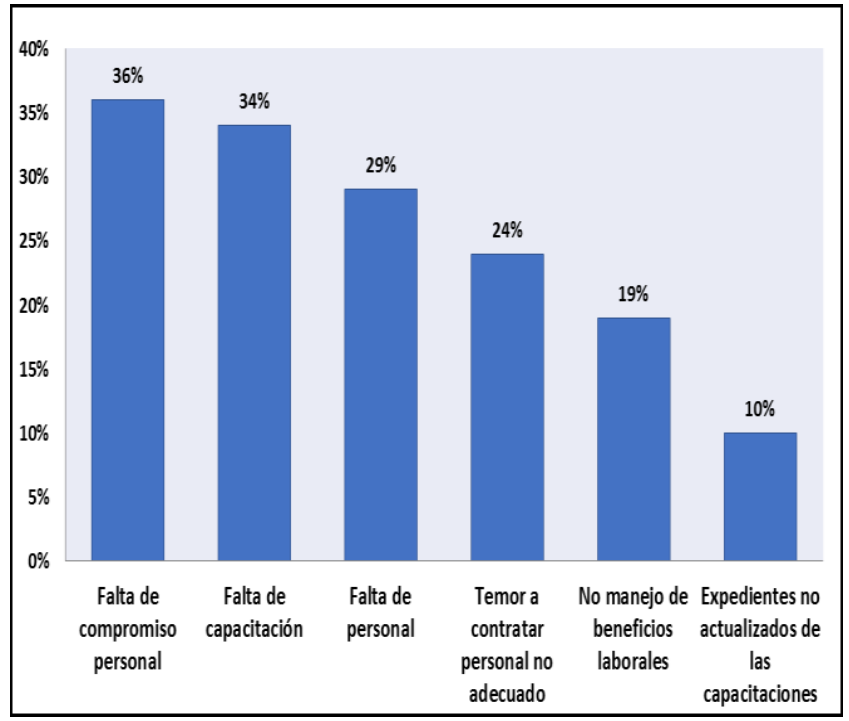

Figura 2. Problemas más comunes que enfrentan con su recurso humano

\subsection{Aplicación de certificaciones, sistemas de inocuidad y calidad}

En la tabla 4 se observa, según los datos obtenidos luego de realizar la encuesta, de 100 pymes encuestadas (julio, 2018), el $68 \%$ sí aplica las BPM, mientras que el $32 \%$ no lo hace. El $60 \%$ de las pequeñas empresas aplican el sistema HACCP, mientras que el $40 \%$ de las pymes indican que no lo implementan, y pertenecen a los diferentes rubros de las empresas agroindustriales evaluadas.

Según datos de un informe sobre la caracterización de empresas agroindustriales alimentarias de Panamá en el año 2013 [2]., de 2.376 empresas agroalimentarias registradas en Panamá se analizó y encuestó a 273 empresas donde el $96 \%$ de las mismas eran pymes. Los resultados al analizar la aplicación de las BPM en las mismas fueron que de 272 empresas, el $71 \%$ sí las aplicaba; mientras que el $29 \%$ de las mismas no lo hacía.
Tabla 4. Certificaciones, inocuidad y calidad

\begin{tabular}{|l|c|c|}
\hline \multirow{2}{*}{\multicolumn{1}{c|}{ Tipo de sistema }} & \multicolumn{2}{c|}{ Aplica } \\
\cline { 2 - 3 } & Sí & No \\
\hline CERTIFICACIÓN & $30 \%$ & $70 \%$ \\
\hline BPM & $68 \%$ & $32 \%$ \\
\hline HACCP & $60 \%$ & $40 \%$ \\
\hline
\end{tabular}

Por otro lado, se muestra en la tabla 5 que, 56 de las 100 empresas encuestadas consideraron que las capacitaciones son muy importantes para el cumplimiento de las buenas prácticas de manufactura (de 6 a 10 puntos); mientras que 44 empresas consideró que las mismas no son tan imprescindibles para un buen desempeño de las BPM (1-5 puntos).

Tabla 5. Grado de importancia que el encuestado imparte a las capacitaciones para el cumplimiento de las BPM

\begin{tabular}{|l|c|c|c|c|c|c|c|c|c|c|}
\hline $\begin{array}{l}\text { Grado de } \\
\text { importancia }\end{array}$ & 1 & 2 & 3 & 4 & 5 & 6 & 7 & 8 & 9 & 10 \\
\hline $\begin{array}{l}\text { Respuesta de } \\
\text { encuestado }\end{array}$ & 13 & 8 & 8 & 6 & 9 & 8 & 14 & 14 & 8 & 12 \\
\hline Resultado global & \multicolumn{4}{|c|}{$\begin{array}{c}44 \text { encuestados } \\
\text { respondiero (1-5) }\end{array}$} & \multicolumn{6}{c|}{$\begin{array}{c}56 \text { escuestados } \\
\text { resporon (6-10) }\end{array}$} \\
\hline
\end{tabular}

En la tabla 6 , se presentan algunas de las recomendaciones expuestas por las personas encuestadas. Según la opinión de los encuestados, para que las capacitaciones en BPH se apliquen y se mantengan con el paso del tiempo, se deben brindar capacitaciones de tipo lúdicas, claras, in situ, llevando registros y controles en planta. Se hace énfasis en que las mismas deben ser de forma frecuente, periódicas, y brindando al personal un programa de incentivos laborales.

Tabla 6. Recomendaciones por el personal encuestado para que las capacitaciones en BPH se mantengan con el tiempo

\section{Recomendaciones}

Deben ser periódicas como el mantenimiento de planta.

Brindar las herramientas necesarias.

Contar con cámaras para detectar sus errores.

Mantener las indicaciones claras a la vista del personal.

Recordatorio constante al personal y vigilar cumplimiento

Verificación in situ de que se aplica la capacitación.

Aplicar pruebas diarias de conocimiento en el tema BPH.

Incentivos económicos al personal.

Registros y controles documentados al final del día.

Recordatorio de la misión, visión de la empresa.

Que las capacitaciones sean más prácticas y activas.

Dar seguimiento en todo el proceso.

Que sean más frecuentes con personal idóneo y experto.

Énfasis en las afectaciones al no cumplir las BPH.

Re- alimentación del personal sobre el beneficio de todos 
En la figura 3 se muestran los pasos concretos del modelo propuesto, donde la decisión de implantar el sistema parte de la gerencia, siendo el mismo un compromiso serio, debido a que todo el esfuerzo se encamina hacia el cumplimiento de los objetivos de calidad e inocuidad, sin dejar de lado la rentabilidad de la empresa. Una vez tomada la decisión de implantar el SGIA, se define la política de calidad e inocuidad de acuerdo a las necesidades de la empresa. Posterior a esta política se conforma un equipo de trabajo multidisciplinario. Debido a que el éxito de este sistema es el resultado del trabajo en equipo y del compromiso de cada integrante, se organiza la investigación de las oportunidades de mejora en materia de inocuidad, como también del recurso humano en cuanto al clima organizacional.

Más adelante se les reúne e inicia el proceso de las capacitaciones lúdicas en cultura de inocuidad. Se evalúan los resultados y se traza la ruta a seguir, siendo de vital importancia una comunicación directa entre los diferentes niveles jerárquicos. Una de las primeras actividades del equipo humano responsable de la implantación del sistema, es elaborar el cronograma para la expansión o transferencia de los procedimientos a seguir, en todos los departamentos de la organización, lo cual permitirá dar un mejor seguimiento y verificación de los resultados, para evaluar el grado de avance y sostenimiento del SGIA.

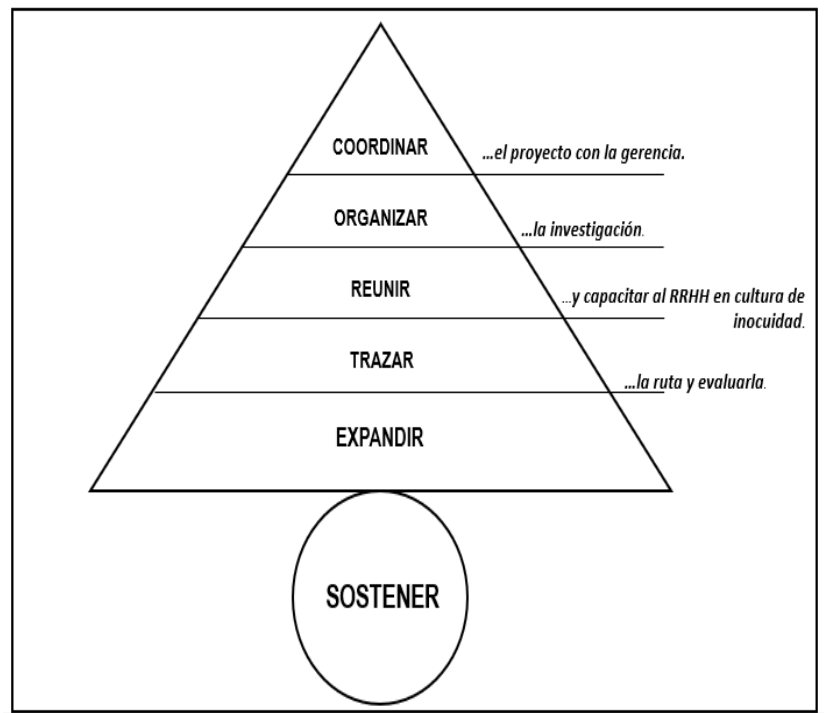

Figura 3. Pasos concretos para la implantación de un SGIA en pymes procesadoras de alimentos en Panamá.

\section{Conclusión}

Todos los actores del proceso son de vital importancia, ya que en una pyme, la influencia que unos a otros ejercen es crucial.

Una de las grandes fallas en la implantación de sistemas de gestión de inocuidad ha sido la falta de sensibilidad para con el personal, pues no se toma en cuenta el clima organizacional.

La capacitación debe ser de carácter permanente en las pymes de Panamá, sobre todo, con actividades lúdicas, así quedan mucho más claros los conceptos, ya que se basan en la cultura de inocuidad propia de la empresa.
Las capacitaciones lúdicas permitieron que el personal en la empresa de estudio estableciera nuevas relaciones con sus compañeros de labor, favoreciendo el cumplimiento de las Buenas prácticas de manufactura.

La falta de compromiso personal, deficiencia en las capacitaciones en materia de inocuidad y la falta de personal fueron los tres principales problemas que enfrentaron con el recurso humano en las pymes de alimentos encuestadas.

La triangulación metodológica como metodología de investigación facilitó el fortalecimiento de las conclusiones obtenidas en el estudio de caso, sobre todo en relación con el establecimiento de pasos concretos, enfocados a minimizar los principales inconvenientes que atraviesa el recurso humano, en las pymes de alimentos en Panamá.

\section{Agradecimiento}

Se agradece a los encuestadores y representantes de las pymes en Panamá por su colaboración, especialmente a la profesora Onesis Vásquez D. L., quien coordinó las encuestas en Azuero.

Agradecimiento al programa Doctoral de Ingeniería de Proyectos de la UTP, pues durante este programa se desarrolló el presente estudio investigativo, periodo 2013-2018.

D.O.C. agradece a la Agencia Chilena para la Inocuidad Alimentaria por sus valiosos aportes e información compartida en el concepto de cultura de inocuidad.

\section{Referencias}

[1] F. Yiannas, "Cultura de Inocuidad Alimentaria, Microbiología de los Alimentos e Inocuidad Alimentaria." New York: Springer Science+Business Media LLC, 2009. http://doi: 10.1007/978-0-387-72867-4.

[2] W. Tejedor, Y. Pittí, V. Guillén, "Caracterización de Empresas Agroindustriales Alimentarias de Panamá," Univ. Tecnológica de Panamá, Centro de Producción e Investigaciones Agroindustriales, pp. 81-94, 2013.

[3] Organización de las Naciones Unidas para la Agricultura y la Alimentación (ONU), Sistemas de Calidad e Inocuidad De Los Alimentos - Manual De Capacitación, Roma, Grupo Editorial Dirección de Información de la FAO, 2002, ISBN 92-5-304115-3.

[4] FAO/OMS, Comité del Códex Alimentarius, Normas Alimentarias,Textos Básicos sobre Higiene de los Alimentos, 2nd., Ed. Roma, Italia, 1997

[5] M. Charalambus, P. Fryer, S. Panayides, M. Smith, "Implementation of Food Safety Management System in small Food businesses in Cyprus," Food Control, vol.57, pp.70-75, 2015. http://doi:10.1016/j.foodcont.2015.04.004

[6] G. Dessler, Administración de Personal, 5ta ed., Ed. México: Pearson Prentice Hall, 2001.

[7] C. Celaya, "Evaluación de la implementación del sistema de análisis de peligros y puntos de control crítico (APPCC) en las pequeñas industrias alimentarias de la comunidad de Madrid", Tesis doctoral, Universidad Complutense de Madrid, Sep. 2004.

[8] Código Sanitario. Ley 66, Gaceta Oficial 10467, Ministerio de Salud, República de Panamá, 10 de noviembre de 1947.

[9] Decreto Ejecutivo 65, Gaceta Oficial 23563, Ministerio de Salud, República de Panamá, 9 de junio de 1997.

[10] Decreto Ejecutivo 352, Gaceta Oficial 24411, Ministerio de Salud. República de Panamá, 10 de noviembre de 2001.

[11] Decreto Ejecutivo 81, Gaceta Oficial 24774, Ministerio de Salud. República de Panamá, 31 de marzo de 2003.

[12] Decreto Ejecutivo 1784, Gaceta Oficial 24774, Ministerio de Salud, República de Panamá, 31 de marzo de 2003.

[13] Comisión Nacional Consultiva de Calidad e Inocuidad de Alimentos, Guía técnica de Buenas Práctica de Manufactura (BPM), Procedimientos Operacionales Estándar de Saneamiento (POES), 2012.

Prisma Tecnológico | Vol. II, n. I, edición 2020. 
[14] D. Watson, S. Pandi, J. Husband, F. Tekelas, "BREXIT and the Implications of Food Safety Cultural Compliance in the Food Manufacturing Sector," Acta Scientific Microbiology, vol 1, pp. 54-62, 2018. http://doi: 10.31080/ASMI.2018.01.0039.

[15] ACHIPIA, Guía para el diseño, desarrollo y aplicación de los Procedimientos Operacionales Estandarizados (POE-SOP). PNI. Agencia Chilena para la Inocuidad y Calidad Alimentaria. Chile, 2018.

[16] ACHIPIA, Guía para el diseño, desarrollo e implantación de los Procedimientos Operacionales Estandarizados de Sanitización (POESSSOP). PNI. Agencia Chilena para la Inocuidad y Calidad Alimentaria. Chile, 2018.

[17] F. Yiannas, Food Safety $=$ Behaviour. 30 técnicas probadas para mejorar el cumplimiento de los empleados, 2018.

[18] R. Larsen, L. M. Morris, An Introduction to Mathematical Statistics and its Applications, 15th ed. Ed. New York, U.S.A.: Prentice Hall, 2017

[19] Ministerio de Desarrollo Agropecuario. (2016) Página web Estadísticas. [Online]. Disponible en: https://www.mida.gob.pa/direcciones/direcciones_ nacionales/direcci-n-de-agro-industrias/estad-sticas.html.
[20] A. Holmes, B. Illoswky y S. Dean, Introductory Business Statistics, Houston: OpenStax, 2018.

[21] J. Rositas, "Los tamaños de las muestras en encuestas de las ciencias sociales y su repercusión en la generación del conocimiento," Innovaciones de Negocios, vol. 11(22), pp 235 - 268, Nov. 2014.

[22] T. Alzás, L. Casa, R. Luengo, J. Torres, S. Verissimo, "Revisión metodológica de la triangulación como estrategia de Investigación," Investigación Cualitativa de Ciencias Sociales, vol. 3, pp. 640-641, 2016.

[23] E. Devaki, "Actividades Lúdicas en la Capacitación, Estudio realizado en una empresa dedicada a la venta de materiales de construcción y rediseño de interiores," Tesis. Universidad de Landívar, Guatemala, 2012.

[24] Naciones Unidas, "Clasificación Industrial Internacional Uniforme de todas las actividades económicas (CIIU)," Departamento de Asuntos Económicos y Sociales, Nueva York pp. 89-95, 2009.

[25] International Labour Office, "Training package on workplace risk assessment and management for small and médium-sized enterprises," Geneva, Switzerland, 2013 